\title{
Are Suprapapillary Biliary Stents Superior to Transpapillary Biliary Stents?
}

\author{
Dániel Pécsi ${ }^{1}$ Áron Vincze ${ }^{2}$ \\ Published online: 17 January 2020 \\ ○) Springer Science+Business Media, LLC, part of Springer Nature 2020
}

\begin{abstract}
Although the duration of patency of biliary stents has been significantly prolonged by the introduction of self-expanding metal stents (SEMS), stent occlusion still remains a major concern. Generally, transpapillary stent placement, where the distal end of the stent traverses the sphincter of Oddi, is favored. The mechanism of stent occlusion is likely related to several factors, including stent-related factors (e.g., diameter, shape, material, side holes) and external factors (bacterial adherence and deposition of dietary fibers into the stent lumen) [1]; the latter may be partially addressed by utilizing stents with anti-reflux mechanisms. In a recent randomized trial, such stents prolonged patency and reduced the incidence of ascending cholangitis [2]. Maintaining the function of the sphincter of Oddi by placing the stent above the sphincter (i.e., suprapapillary or intraductally) might be another approach to solving this problem (Fig. 1).
\end{abstract}

In this issue of Digestive Diseases and Sciences, Shin et al. report a retrospective cohort study comparing suprapapillary and transpapillary endoscopic stent placement in patients with hilar cholangiocarcinoma [3]. The main outcomes of the study were stent patency, stent-related adverse events, stent revision success rates, and patient survival. Over a period of 10 years, they identified 73 cases (44 suprapapillary and 29 transpapillary). Stent patency (140 vs. 157 days, respectively; $p=0.732$ ), adverse stent-related events, and patient survival did not differ significantly between groups. Nevertheless, the rate of successful endoscopic revision was much lower in the suprapapillary stent group (25.0\%) compared with the transpapillary stent group (68.8\%). A multivariate analysis indicated that that stent position itself was associated with stent revision failure. The

Áron Vincze

vincze.aron@pte.hu

1 Institute for Translational Medicine, Medical School, University of Pécs, Pécs, Hungary

2 First Department of Medicine, Medical School, University of Pécs, Ifjúság u. 13, Pécs 7624, Hungary high failure rate was explained by greater difficulty in cannula and guide-wire insertion with a suprapapillary approach since cancer tissue or bile sludge blocked the distal part of the stent, a finding not observed with the transpapillary approach. Moreover, the etiology of stent occlusion significantly differed between groups: Stent ingrowth was more frequent in the suprapapillary group $(75.0 \%$ vs. $31.3 \%)$, whereas sludge was more frequent in the transpapillary group $(50.0 \%$ vs. $6.8 \%)$. It is not known whether ingrowth in the suprapapillary group was due to the shorter length of stents applied or to proximal migration. Less frequent sludge formation in the suprapapillary group might be explained by decreased duodeno-biliary reflux. Sphincterotomy, a procedure that by itself promotes duodeno-biliary reflux, was performed in all patients.

Although these results are interesting and worth further exploring, these observations are limited since, as a retrospective single-center study with a relatively small sample size, definitive conclusions regarding the best approach for biliary stenting cannot be made.

Not many studies have compared the suprapapillary and transpapillary stent approaches. Only two randomized controlled trials in patients with malignant biliary obstruction have been published, one using metal stents [4] and the other using plastic stents [5]. Both studies concluded that there was no difference in stent patency between approaches though stent dislocation was more common with suprapapillary stents. In a retrospective study of 152 patients with hilar cholangiocarcinoma managed by bilateral side-by-side metal stents deployed above or across the sphincter, the suprapapillary group experienced similar patency rates but fewer complications [6]. There was considerable heterogeneity in the patient population of these studies as they included patients with both distal and hilar tumors. In contrast to the present study, very few or no patients in the suprapapillary group underwent sphincterotomy (Table 1). As sphincterotomy is not necessary with suprapapillary stent placement, its avoidance would 
Fig. 1 Suprapapillary and transpapillary stent placement

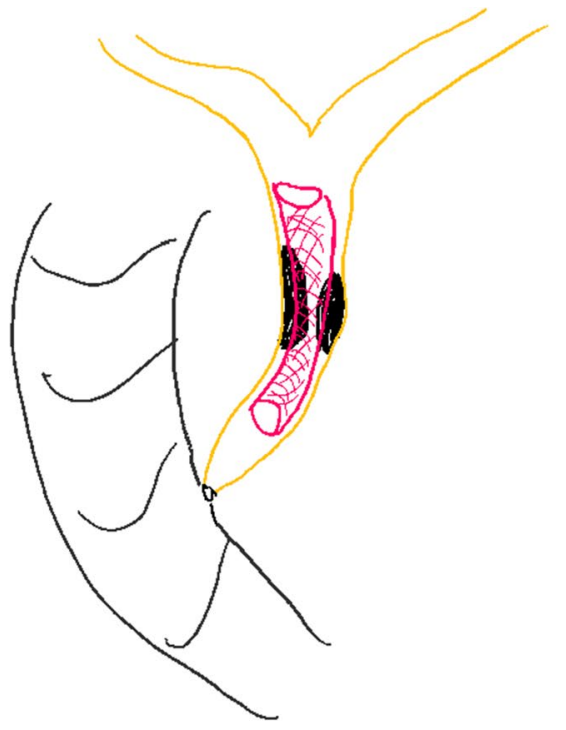

Suprapapillary stent placement

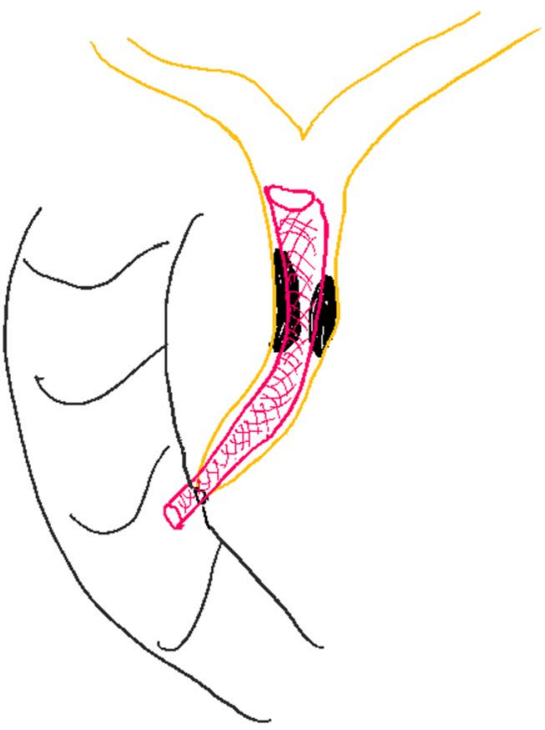

Transpapillary stent placement

Table 1 Summary of studies comparing suprapapillary and transpapillary stent placement

\begin{tabular}{llllllcr}
\hline Author, publication date & Study design & Stent type & Number of & \multicolumn{2}{c}{ Number of patients } & Sphincterotomy \\
\cline { 5 - 6 } & & & centers & Supra & Trans & Supra & Trans \\
\hline Cho et al. (conference abstract) [4] & RCT & FC-SEMS & 12 & 38 & 37 & None & All \\
Cosgrove et al. [6] & Retrospective & U-SEMS & 2 & 52 & 100 & $9.6 \%$ & $90 \%$ \\
Pedersen et al. [5] & RCT & Straight PS 10 Fr & 1 & 17 & 17 & None & None \\
Shin et al. [3] & Retrospective & U-SEMS & 1 & 44 & 29 & All & All \\
\hline
\end{tabular}

$F C$ fully covered, $U$ uncovered, $P S$ plastic stent, $R C T$ randomized controlled trial

eliminate complications associated with sphincterotomy as well as maintain the natural barrier that prevents duodenobiliary reflux.

Regarding stent revision, threads have been added to the distal ends of plastic stents inserted across the papilla in order to facilitate removal [7]. This approach has not been tested, but might be useful, for metal stents as well.

Our previous meta-analysis of three prospective and six retrospective studies $(n=289$ suprapapillary and 325 transpapillary) reported significantly lower rates of stent occlusion, cholangitis, and pancreatitis with suprapapillary stenting compared with transpapillary stenting. This difference was only apparent in patients with plastic stents, while no benefits of suprapapillary stent position were seen in patients with SEMS [8].

Although the authors should be congratulated for reviving this technique, the role of suprapapillary stenting for extrapancreatic malignant biliary obstruction still remains uncertain. Well-designed, multicenter, randomized controlled trials with appropriate sample sizes are needed.
Acknowledgments Open access funding provided by University of Pécs (PTE).

\section{References}

1. Van Berkel A, Van Marle J, Groen A, Bruno M. Mechanisms of biliary stent clogging: confocal laser scanning and scanning electron microscopy. Endoscopy. 2005;37:729-734.

2. Hu B, Wang T, Wu J, et al. Antireflux stents to reduce the risk of cholangitis in patients with malignant biliary strictures: a randomized trial. Endoscopy. 2014;46:120-126.

3. Shin J, Park J-S, Jeong S, Lee DH. Comparison of the Clinical Outcomes of Suprapapillary and Transpapillary Stent Insertion in Unresectable Cholangiocarcinoma with Biliary Obstruction. Dig Dis Sci. (Epub ahead of print). https://doi.org/10.1007/s1062 0-019-05859-1.

4. Cho JN, Han J, Kim HG, et al. Prospective randomized trial comparing covered metal stent placed above and across the sphincter of Oddi in malignant biliary obstruction. Gastrointest Endosc. 2013;77:AB139-AB140.

5. Pedersen FM, Lassen AT, de Muckadell OBS. Randomized trial of stent placed above and across the sphincter of Oddi in malignant bile duct obstruction. Gastrointest Endosc. 1998;48:574-579.

6. Cosgrove N, Siddiqui AA, Adler DG, et al. A comparison of bilateral side-by-side metal stents deployed above and across the 
sphincter of Oddi in the management of malignant hilar biliary obstruction. J Clin Gastroenterol. 2017;51:528-533.

7. Ishiwatari H, Hayashi T, Ono M, Sato T, Kato J. Newly designed plastic stent for endoscopic placement above the sphincter of Oddi in patients with malignant hilar biliary obstruction. Dig Endosc. 2013;25:94-99.

8. Pécsi D, Farkas N, Hegyi P, Márta K, Szakács Z, Vincze Á. Suprapapillary biliary stent placement might have longer latency time compared to transpapillary stent placement-a systematic review and meta-analysis. Endoscopy. 2018;50:S43.

Publisher's Note Springer Nature remains neutral with regard to jurisdictional claims in published maps and institutional affiliations. 